\title{
Ultrasound Features of Palmar Fibromatosis or Dupuytren Contracture
}

\author{
Gregory Morris, MD, Jon A. Jacobson, MD \, Monica Kalume Brigido, MD, Kara Gaetke-Udager, MD, \\ Corrie M. Yablon, MD, Qian Dong, MD
}

Received February 15, 2018, from the Department of Radiology, University of Michigan, Ann Arbor, Michigan USA. Manuscript accepted for publication April 30, 2018.

Address correspondence to Jon A Jacobson, MD, Department of Radiology, University of Michigan, 1500 East Medical Center Dr, TC2910L, Ann Arbor, MI 48109S326 USA.

E-mail: jiacobsn@umich.edu
Objectives - To retrospectively characterize the ultrasound appearance of palmar fibromatosis in patients with a surgical or clinical diagnosis of palmar fibromatosis.

Methods-A search of ultrasound reports from 2005 to 2015 and a subsequent search of medical records were performed to identify patients with a surgical or clinical diagnosis of palmar fibromatosis. The ultrasound images were retrospectively reviewed to record the lesion location, size, echogenicity, compressibility, hyperemia, and calcification.

Results-A total of 36 patients were identified (average age, 60 years; $61 \%$ male), yielding a total of S5 palmar fibromatosis lesions, of which $2 \%, 7 \%, 29 \%, 36 \%, 20 \%$, and $5 \%$ were located at the first, second, third, fourth, and fifth digits and between the fourth and fifth digits, respectively. The lesions were located directly superficial to the flexor tendons in $93 \%$ with their epicenters at the distal metacarpal in $89 \%$. Average lesion dimensions were $13.1 \mathrm{~mm}$ in length, $6.8 \mathrm{~mm}$ in width, and $2.5 \mathrm{~mm}$ in depth. On ultrasound images, the lesions were characteristically hypoechoic (98\%) and noncompressible (95\%). Atypical features included calcification (2\%), compressibility (5\%), hyperemia on color Doppler images (6\%), epicenters at the metacarpophalangeal joint $(7 \%)$ or proximal phalanx $(4 \%)$, and location superficial but lateral to the flexor tendons $(7 \%)$.

Conclusions-Palmar fibromatosis most commonly appears hypoechoic and is located directly superficial to the flexor tendons with an epicenter at the distal metacarpal, most commonly the fourth digit. However, the epicenter location may be at the distal metacarpal and proximal phalanx of other digits, adjacent to the flexor tendons, with possible hyperemia and calcification.

Key Words - Dupuytren contracture; fibromatosis; hand; musculoskeletal; palmar; ultrasound

$\mathrm{P}$ almar fibromatosis, also known as Dupuytren disease or Dupuytren contracture, is a benign fibroproliferative disorder in which subcutaneous fibrous nodules arise within the palmar fascia of the hand, eventually forming cordlike attachments with the adjacent flexor tendons. ${ }^{1,2}$ Progressive shortening of these cordlike attachments ultimately results in debilitating flexion contractures of the fingers. Palmar fibromatosis is common, affecting approximately $20 \%$ of people older than 65 years, and is associated with other fibromatoses, as well as diabetes mellitus, alcoholism, and epilepsy. ${ }^{3}$ Imaging may be used to confirm palmar fibromatosis and for imagingguided treatment. ${ }^{4}$

Magnetic resonance imaging has been shown to accurately detect palmar fibromatosis, appearing as subcutaneous nodules and cords located superficial and parallel to the flexor tendon. ${ }^{5}$ Although 
ultrasound has been used to guide percutaneous collagenase injections of palmar fibromatosis, the ultrasound appearance of palmar fibromatosis has not been fully described in the literature.

In our clinical practice, ultrasound is routinely used to assess hand and wrist disorders, including palmar fibromatosis. We have found variable locations, as well as grayscale and color Doppler appearances. The purpose of this study was to retrospectively characterize the ultrasound appearance of palmar fibromatosis in patients with a surgical or clinical diagnosis of palmar fibromatosis.

\section{Materials and Methods}

The Institutional Review Board approved our study, and informed consent was waived. A retrospective search of the radiology database for ultrasound reports from 2005 to 2015 was performed using the key words "Dupuytren" and "contracture" and the key phrases "palmar fibromatosis" and "palmar fibroma." Medical records were then reviewed to determine whether a

Figure 1. Images from a 68-year-old woman with palmar fibromatosis. Uitrasound images long axis (A) and short axis (B) to the flexor tendons (T) of the fourth digit show hypoechoic palmar fibromatosis (arrows) at the level of the distal metacarpal (MC).
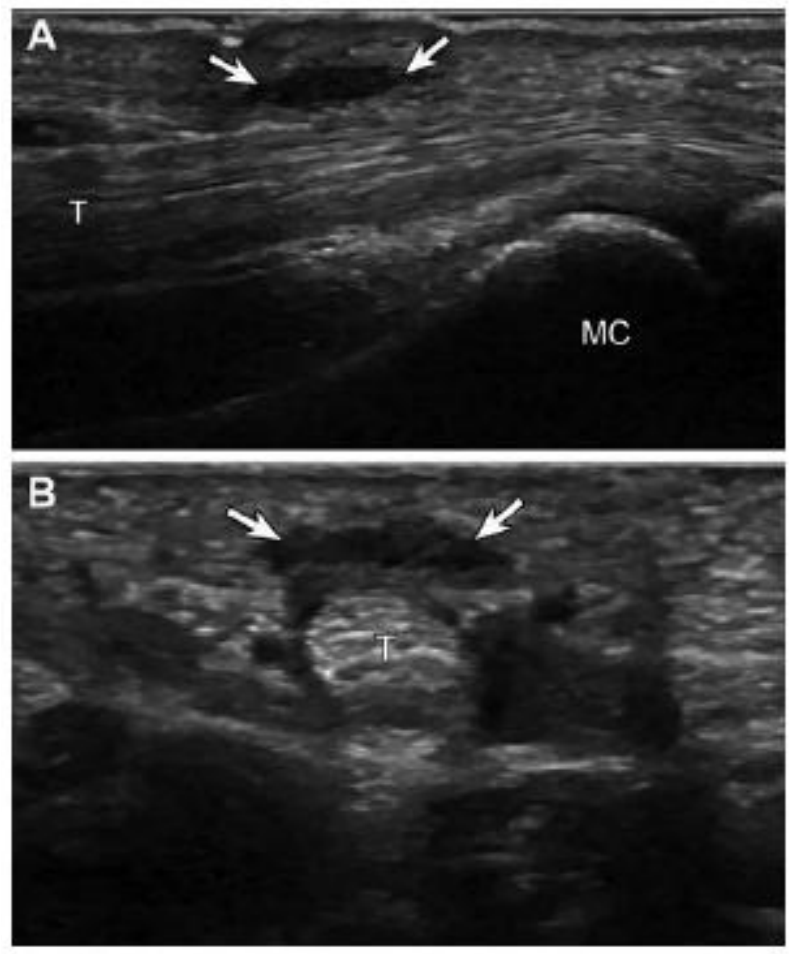

clinical or surgical diagnosis of palmar fibromatosis was present. Clinical findings of palmar fibromatosis included palpable firm superficial nodules or cords, possible tenderness, involving the palmar aspect of the hand, with possible flexion of the digits with an inability to place the palm flat on a tabletop (the Hueston tabletop test). Other clinical information, including histories of diabetes, epilepsy, alcoholism, plantar fibromatosis, Peyronie disease, and adhesive capsulitis, was recorded. Patients were excluded if they had no clinical or surgical diagnosis of palmar fibromatosis, or if they had previously received treatment for palmar fibromatosis before the ultrasound examinations.

Ultrasound reports and images including cine clips were retrospectively reviewed in consensus by 2 authors with 4 and 22 years of experience, and the following information was recorded: location of the abnormality

Figure 2. Images from a 67 -year-old woman with palmar fibromatosis. Ultrasound images long axis (A) and short axis (B) to the flexor tendons ( $\mathrm{T}$ ) of the third digit show hypoechoic palmar fibromatosis (arrows) at the level of the distal metacarpal (MC). Note the lack of flow on the color Doppler image in $\mathbf{B}$.
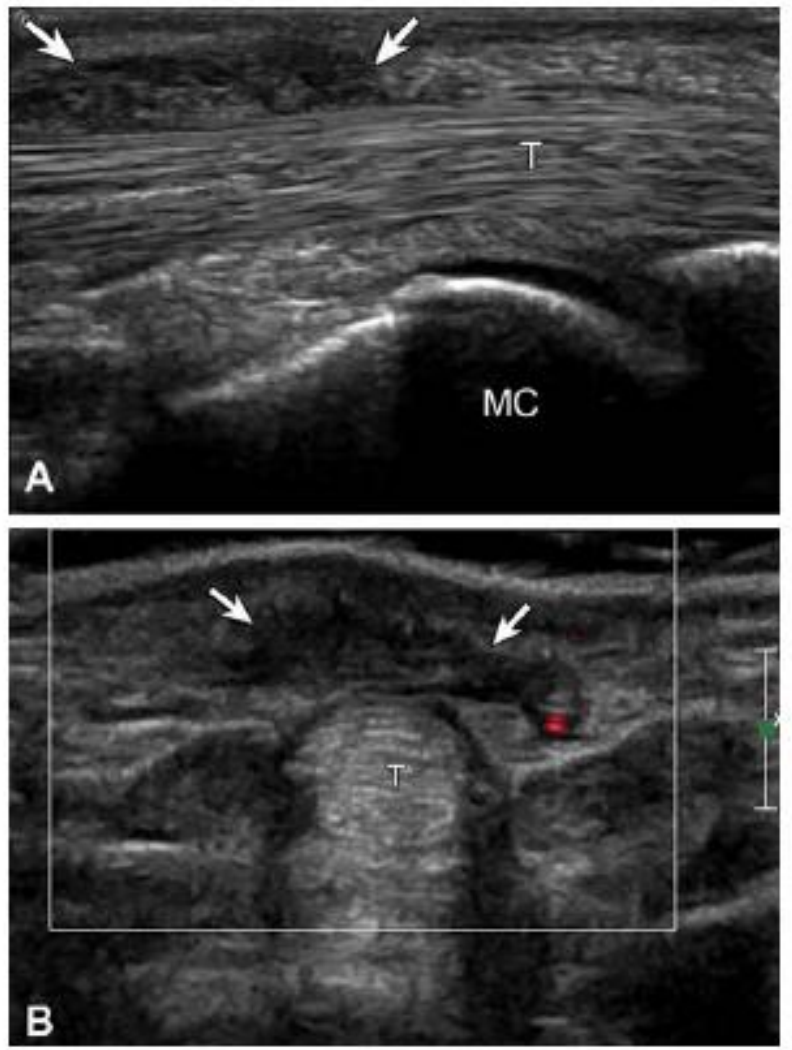
(which digit, epicenter relative to the long axis of a digit, and whether the abnormality was superficial to the tendon), size (length, width, and depth), echogenicity (anechoic, hypoechoic, isoechoic, hyperechoic, or heterogeneous relative to adjacent subcutaneous tissues), hyperemia on conventional color or power Doppler imaging (present or absent), compressibility (present or absent), and calcification (defined as a hyperechoic focus with possible shadowing, present or absent). Patient demographics were also recorded (age, sex, and side).

\section{Results}

The initial key word search identified 51 patients, and 15 were excluded because there was no clinical or surgical diagnosis of or there was prior treatment for palmar fibromatosis. The final group consisted of 36 patients, comprising $61 \%$ (22 of 36) male and $39 \%$ (14 of 36) female patients. The average age of all patients was 60 years (range, 36-79 years). The average age of the male patients was 58 years (range, 36-79 years), and the average age of the female patients was 63 years (range, $51-70$ years). In $8 \%$ (3 of 36) of patients, there was bilateral involvement, resulting in a total of 39 hand ultrasound examinations for review, including 23 of the right hand and 16 of the left hand. The history provided at the time of imaging included an assessment of a palpable abnormality, confirmation of suspected palmar fibromatosis, or both.

A total of 55 palmar fibromatosis lesions were identified on the ultrasound images, of which $2 \%$ ( 1 of 55) were located within the first digit, $7 \%$ (4 of 55) within the second digit, $29 \%$ (16 of 55) within the third digit, $36 \%$ ( 20 of 55 ) within the fourth digit, $20 \%$ (11 of 55) within the fifth digit, and $5 \%$ ( 3 of 55) between the fourth and fifth digits. There was involvement of only 1 digit in $62 \%$ ( 24 of 55) of ultrasound images, 2 digits in $15 \%$ ( 8 of 55), and 3 digits in 7\% ( 4 of 55 ).

The lesions showed a hypoechoic appearance in $98 \%$ (54 of 55; Figures 1 and 2) and a predominant isoechoic appearance in $2 \%$ ( 1 of 55; Figure 3 ). No lesions showed an anechoic or a hyperechoic appearance. Hyperemia was shown in 6\% (3 of 54) and was absent in $94 \%$ (51 of 54). Intralesional calcification was observed in $2 \%$ ( 1 of 55). Noncompressibility of the lesion, as recorded on still images or cine clips, was demonstrated in $95 \%$ ( 52 of 55) and compressibility in $5 \%$ ( 3 of 55). The lesion epicenter was located in the region of the distal metacarpal in $89 \%$ ( 49 of 55), in the region of the metacarpophalangeal joint in 7\% (4 of 55; Figure 4), and in the region of the proximal phalanx in

Figure 3. Images from a 50-year-old man with palmar fibromatosis. Ultrasound images long axis to the third digit (A), long axis to the fourth digit (B), and short axis to the third and fourth digits (C) show predominantly iscechoic and hypoechoic palmar fibromatosis (cursors and arrows). Ultrasound image long axis to fith digit (D) shows an additional nodule (cursors) just distal to the proximal interphalangeal joint (PIP). DIP indicates distal interphalangeal joint; $L$, lumbrical muscles; MC, metacarpal; MP, middle phalanx; and $T$, flexor tendons.
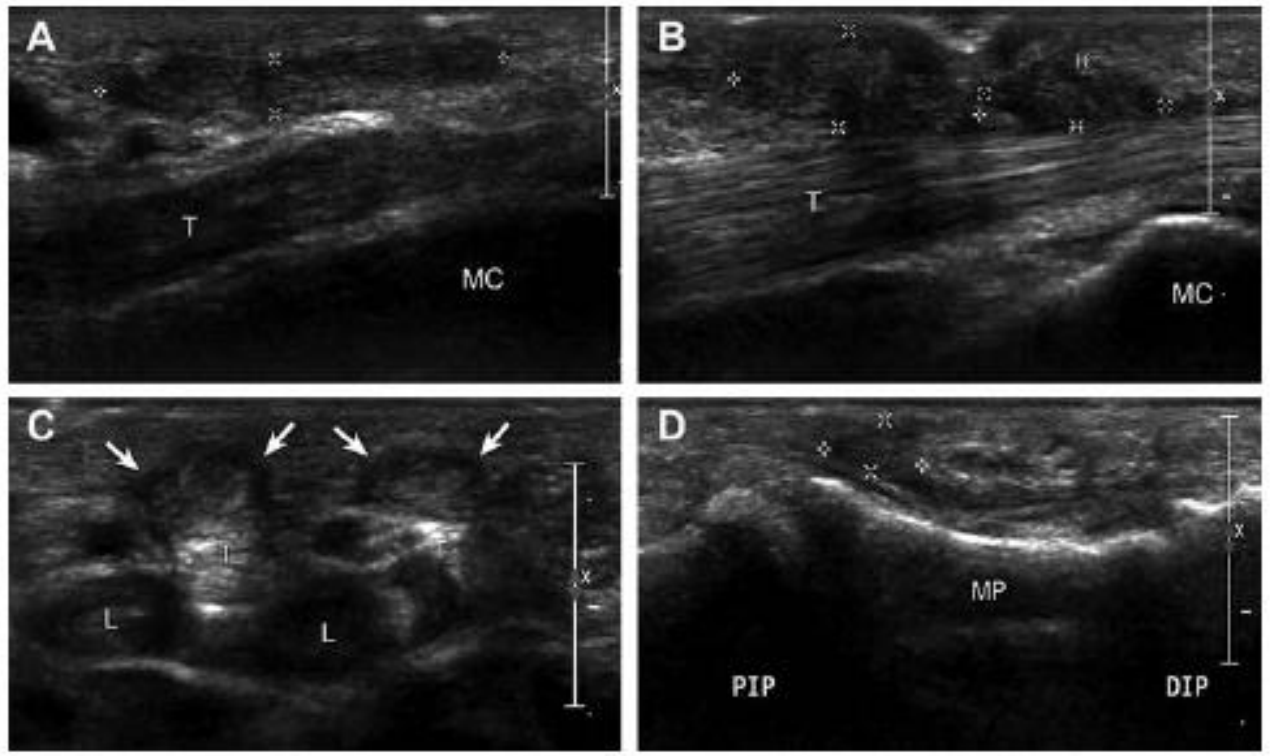
4\% ( 2 of 55; Figure 3D). The lesions were located directly superficial to the flexor tendons in $93 \%$ ( 51 of 55 ) and superficial but lateral to the flexor tendons in

Figure 4. Images from a 61-year-oid man with palmar fibromatosis. Ultrasound images long axis $(\mathbf{A})$ and short axis (B) to the flexor tendons $(T)$ of the fourth digit show hypoechoic paimar fibromatosis (arrows) extending beyond the distal metacarpal (MC) over the metacarpophalangeal joint.
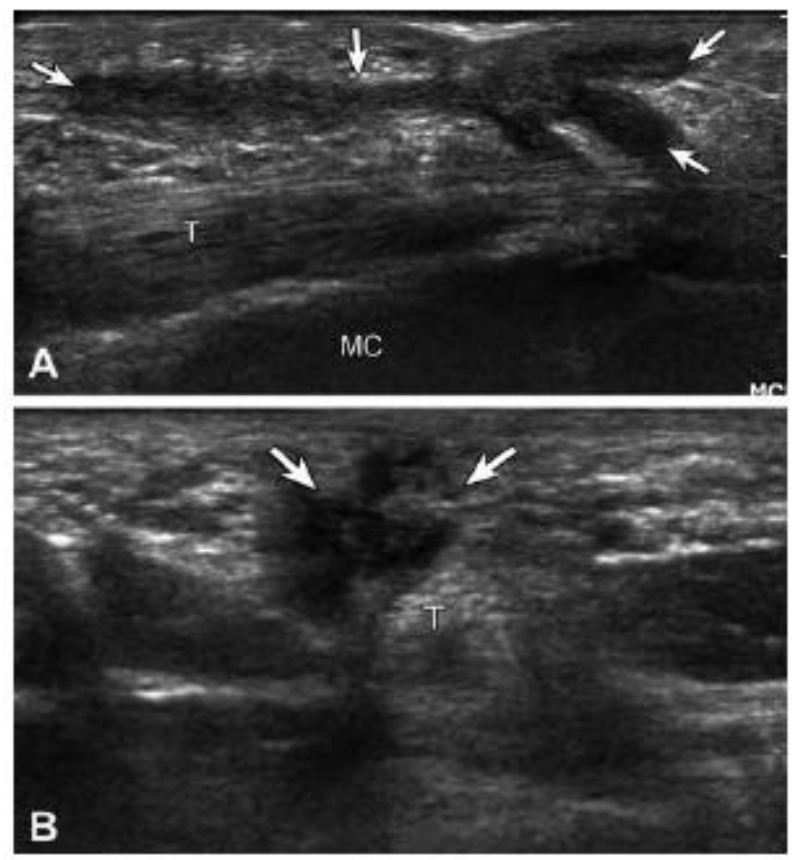

$7 \%$ (4 of 55). The average length of the lesions measured $13.1 \mathrm{~mm}$ (range, $4.1-33.5 \mathrm{~mm}$ ); the average width measured $6.8 \mathrm{~mm}$ (range, $3.0-19.0 \mathrm{~mm}$ ); and the average depth measured $2.5 \mathrm{~mm}$ (range, $0.7-4.6 \mathrm{~mm}$ ).

Of the 36 patients, all had a clinical diagnosis of palmar fibromatosis, and 19\% (7 of 36) had surgical and histologic correlations as part of the inclusion criteria. One of these patients with surgical confirmation also had magnetic resonance imaging (Figure 5). Surgical findings described a mass or nodule, and pathologic findings described fibrous tissue, consistent with the diagnosis. A concurrent diagnosis of diabetes was identified in $14 \%$ (5 of 36), plantar fibromatosis in $6 \%$ ( 2 of 36 ), Peyronie disease in $9 \%$ ( 2 of 22 male patients), adhesive capsulitis in 11\% (4 of 36), and alcoholism in 3\% ( 1 of 36). A concurrent diagnosis of epilepsy was not identified in any of the patients. Of the 15 patients excluded from this study without a clinical or surgical diagnosis of palmar fibromatosis, 1 patient had a ruptured epidermoid cyst at surgery (Figure 6).

\section{Discussion}

Ultrasound may be used to confirm palmar fibromatosis and for imaging-guided treatment; however, to our knowledge, the ultrasound appearance of palmar fibromatosis has yet to be fully described in the literature. The results of our study show that palmar fibromatosis

Figure 5. Images from a 48-year-old man with palmar fibromatosis. Ultrasound images long axis (A) and short axis (B) to the flexor tendons (T) of the third digt show hypoechoic palmar fibromatosis (arrows). MC indicates metacarpal head. T2-weighted fat saturation (C) and T1-weighted fat saturation post-intravenous gadolinium (D) magnetic resonance images show enhancing mixed but predominately low-signal palmar fibromatosis (arrows) superticial to the third-digit flexor tendons.
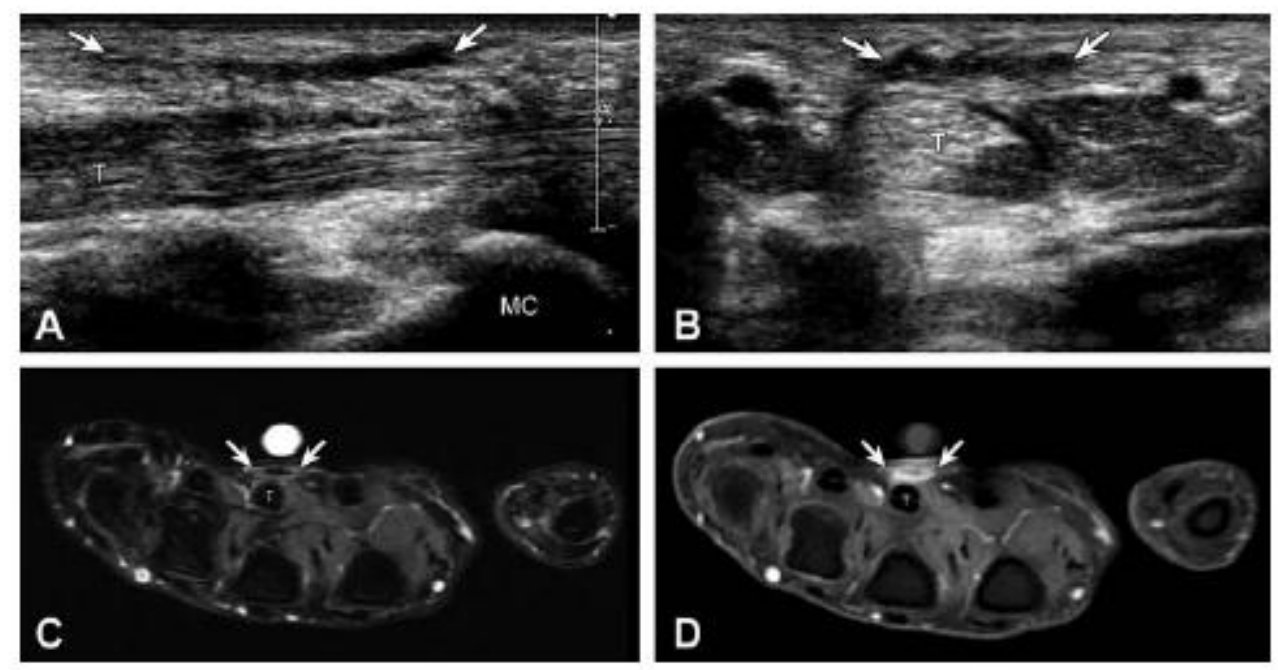
most commonly appears hypoechoic and is located directly superficial to the flexor tendons with an epicenter at the distal metacarpal, most commonly the fourth digit. Uncommonly, the epicenter location may be at the distal metacarpal and proximal phalanx of other digits, adjacent to the flexor tendons, with possible hyperemia and calcification.

Palmar fibromatosis is a benign fibroproliferative disorder in which subcutaneous fibrous nodules arise within the palmar fascia of the hand. ${ }^{1,2}$ Debilitating flexion contractures of the fingers result from progressive shortening of cordlike attachments between these nodules and the adjacent flexor tendons. Histologically, the early proliferative phase of the disease features hypercellular nodules composed of fibroblasts, whereas older end-stage lesions are, by comparison, less cellular and show increased collagen content. ${ }^{3}$

Although an underlying genetic predisposition and a propensity for palmar fibromatosis to affect white people of Northern European descent is supported by twin and family studies, a multifactorial etiology is suggested by additional studies implicating trauma, microvascular injury, and immunologic processes. ${ }^{3}$ Palmar fibromatosis

Figure 6. Images from a 45-year-old man with a nptured epidermoid cyst. Extended field-of-view ultrasound images long axis (A) and short axis (B) to the flexor tendons ( $\mathrm{T}$ ) show a mixed-echogenicity but predominantly hypoechoic ruptured epidermoid cyst (arrows).
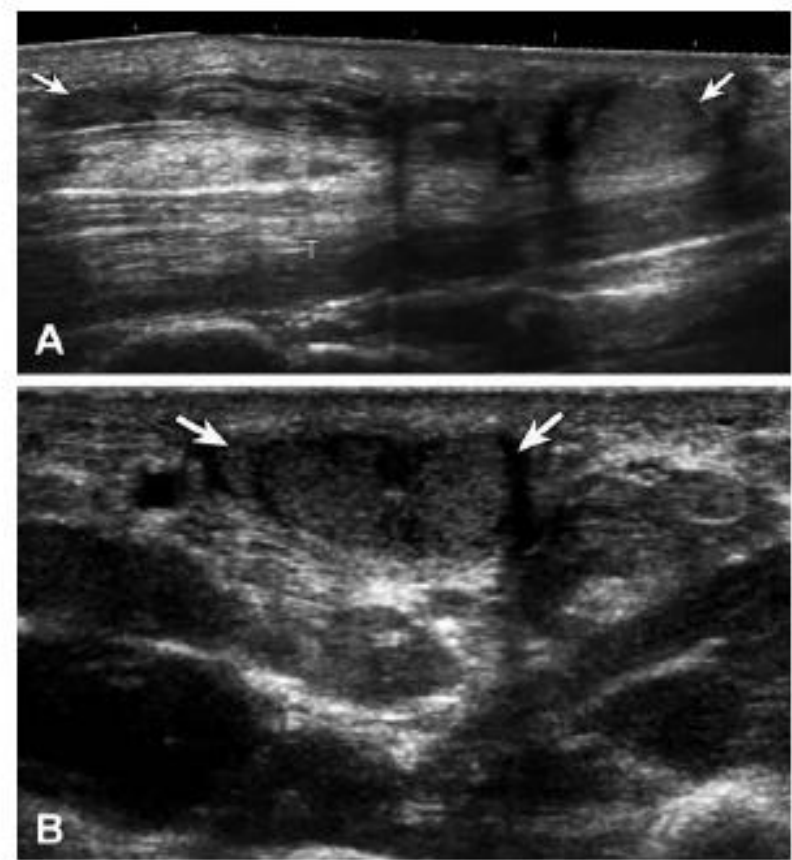

may be seen concurrently in the setting of other fibromatoses such as plantar fibromatosis, Peyronie disease, knuckle pads, and adhesive capsulitis. ${ }^{2,3,6}$ Associations with diabetes mellitus types 1 and 2, epilepsy, and alcoholism have also been described. ${ }^{2,3}$ In our study, a retrospective review of the patients' medical records revealed concurrent diagnoses of diabetes (14\%), plantar fibromatosis (6\%), Peyronie disease (9\% of the male patients), adhesive capsulitis (11\%), and alcoholism (3\%). However, a concurrent diagnosis of epilepsy was not identified in any of the patients.

In our study, palmar fibromatosis lesions most commonly were found within the fourth digit, followed by the third, fifth, second, and first digits in decreasing order of frequency. The lesions were typically located directly superficial to the flexor tendons (93\%) with their epicenter in the region of the distal metacarpal (89\%). The lesions were characteristically hypoechoic (98\%) and noncompressible (95\%). Atypical features included intralesional calcification (2\%), compressibility (5\%), and hyperemia on color Doppler images (6\%). Lesion epicenters located more distally than what was typical were observed in the region of the metacarpophalangeal joint (7\%) and the the proximal phalanx (4\%). In 4 patients $(7 \%)$, the lesions were located superficial but lateral to the flexor tendons, rather than directly superficial to them.

Several benign and malignant lesions in the hand may simulate palmar fibromatosis, including epithelioid sarcomas, giant cell tumors of the tendon sheath, ganglion cysts, inclusion cysts, stenosing tenosynovitis without triggering, edematous changes of the hand, and thickening and callus formation related to occupational activity. ${ }^{2}$ Familiarity with the imaging features of palmar fibromatosis can aid in distinguishing this disease from these various other entities. For example, a hypoechoic mass superficial to a flexor tendon of the third, fourth, or fifth digit at the level of the distal metacarpal, where the length of the lesion is greater than its width, is typical for palmar fibromatosis and helps exclude other diagnoses. Knowledge of the ultrasound appearance of palmar fibromatosis is also needed if ultrasound guidance is used for collagenase injection therapy to also avoid complications associated with injections into tendons, neurovascular structures, and collagen-containing structures within the hand. ${ }^{7}$

One limitation of our study was its retrospective design, which restricted our ultrasound imaging review 
to the existing images; however, the presence of cine clips complementing the static images added valuable real-time information. Another limitation was lack of surgical confirmation in all cases. When surgical and histologic data were not present, only patients in whom unequivocal clinical evidence for palmar fibromatosis were included. Another limitation was our small sample size, which was likely secondary to patients with palmar fibromatosis who may not have undergone imaging. The clinical importance of the atypical ultrasound findings of palmar fibromatosis was outside the scope of this study but warrants further investigation.

In conclusion, palmar fibromatosis most commonly appears hypoechoic and is located directly superficial to the flexor tendons with an epicenter at the distal metacarpal, most commonly involving the fourth digit. However, the epicenter location may be at the distal metacarpal and proximal phalanx of other digits, adjacent to the flexor tendons, with possible hyperemia and calcification. Knowledge of both the common and atypical ultrasound appearances of palmar fibromatosis can improve diagnostic accuracy and avoid potential complications associated with percutaneous therapies.

\section{References}

1. Robbin MR, Murphey MD, Temple HT, Kransdor Ml, Choi JJ. Imaging of musculoskeletal fibronatosis. Radiogmaphics $2001 ; 21: 585-600$.

2. Rayan GM. Dupuytren disease: anatomy, pathology, presentation, and treatment. J Bowe Jow Sarg Am 2007; 89:189-198.

3. Murphey MD, Ruble CM, Tyszko SM, Zbojniewicz AM, Potter BK, Miettinen M. From the anchives of the AFIP: musculoskeletal fibromatoses - radiologic-pathologic conclation Radiographics 2009; 29:2143-2173.

4. Sampson S, Meng M, Schulte A, Trainor D, Montenegro R, Aufiero D. Management of Dupuytren contracture with ultrasound-guided lidocaine injection and needle aponeurotomy coupled with osteopathic manipulative treatment. / An Ostoppath Assoc 2011; 111:113-116.

5. Yacoe ME, Bergman AG, Ladd AL, Hellman BH. Dupuytren's contracture: MR imaging findings and correlation between MR signal intensity and celluhrity of lesions. A/R Am J Roostgenol 1993; 160:813-817.

6. Smith SP, Devaraj VS, Bunker TD. The association between frozen stwoulder and Dupuytren's disease. I Shoulder Ellow Surg 2001; 10: 149-151.

7. Badalamente MA, Hurst LC, Benhaim P, Cohen BM. Efficacy and safety of collagenase clostridium histolyticum in the treatment of proximal interphalangeal joints in Dupuytren contracture: combined analysis of four phase 3 clinical trials. J Howd Surg An 2015; 40:975-983. 